\title{
IRA Code Design for IDMA-based Multi-Pair Bidirectional Relaying Systems
}

\author{
F. Lenkeit, C. Bockelmann, D. Wübben, A. Dekorsy \\ Department of Communications Engineering \\ University of Bremen, Germany \\ Email: \{lenkeit, bockelmann, wuebben, dekorsy\}@ant.uni-bremen.de
}

\begin{abstract}
In this paper, the design of Irregular Repeat Accumulate (IRA) Codes for Interleave-Division Multiple-Access (IDMA)-based multi-pair bidirectional relaying systems is presented. Bidirectional relaying allows to efficiently cope with the loss in spectral efficiency that is inherent to relaying systems due to the half-duplex constraint. However, as soon as multiple communication pairs use one common relay for communication, the question of medium access arises. Since orthogonal medium access schemes again introduce a rate loss to the system, nonorthogonal medium access based on IDMA is proposed. Further, in order to optimize the IDMA-based system regarding spectral efficiency, IRA codes are applied which allow for a highly flexible code design. This paper gives a description of the general code design process based on EXIT-analysis. Moreover, some design examples are given and the resulting systems are compared to conventionally coded IDMA systems based on convolutional codes.
\end{abstract}

\section{INTRODUCTION}

Relaying has been an extensively investigated research topic over the last decades as it allows to cope with path-loss and fading in mobile radio systems. The main drawback of relaying, however, is the loss in spectral efficiency due to the half-duplex constraint. To overcome this drawback, bidirectional relaying has been investigated considering both transmission directions between two nodes jointly. Applying techniques like physical layer network coding (PLNC) [1] in the first phase, where both nodes transmit to the relay, and network coding (NC) [2] [3] in the second phase, where the relay boadcasts to both nodes, the overall number of required transmission slots is reduced and, thus, the spectral efficiency is increased.

As soon as one relay supports multiple communication pairs, the question of medium access arises. In the literature usually orthogonal medium access is assumed during both transmission phases. This, however, introduces a significant control signaling overhead, especially in larger networks. For heterogenous networks with asymmetric rate requirements, this overhead might increase even more. To this end, it seems resonable to combine bidirectional relaying with nonorthogonal medium access. A first approach to use common CDMA for this purpose has been presented in [4]. Here, both

This work has been performed in the framework of the FP7 project ICT317669 METIS, which is partly funded by the European Union. The authors would like to acknowledge the contributions of their colleagues in METIS, although the views expressed are those of the authors and do not necessarily represent the project.

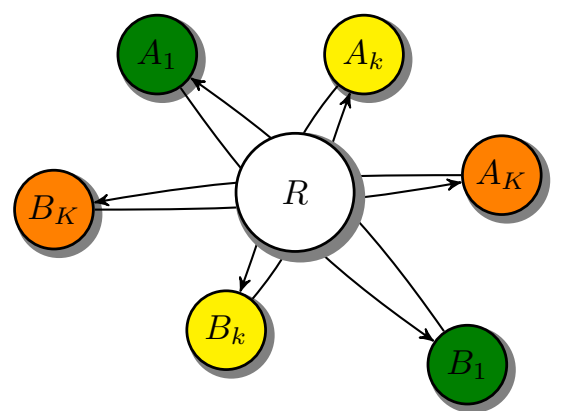

Fig. 1. Topology of the considered multi-pair bidirectional relaying system with $K$ communication pairs and one common relay $R$.

nodes of one communication pair use the same spreading sequence allowing the relay to estimate both messages jointly. Another non-orthogonal access scheme which adopts many advantages of CDMA is Interleave-Division Multiple-Access (IDMA) [5]. In contrast to CDMA, for IDMA channel coding and spectral spreading are combined, devoting the complete bandwidth expansion to coding. The only means to distinguish the different users are user specific interleavers. IDMA has been shown to clearly outperform CDMA under practical considerations in terms of performance and complexity in [6]. This is especially true for overloaded systems which have to be considered when aiming at high spectral efficiencies.

One of the most important aspects of IDMA is the applied low-rate channel code. A very common choice is the serial concatenation of a convolutional code and a repetition code as a compromise between error correcting capabilities and decoding complexity. However, as can be shown by EXITanalysis [7] there is usually still a significant rate loss for this code combination. Thus, in order to optimize the system throughput, the channel code should be thoroughly designed for the particular working point. A suitable class of highly flexible codes with a low encoding and a moderate decoding complexity are Irregular Repeat Accumulate (IRA) codes [8]. These codes have firstly been applied to iterative detection schemes in [9] where IRA codes were designed in the context of a MIMO system.

In this paper, we apply IRA codes to multi-pair bidirectional relaying systems based on IDMA. We describe the principal design process and give concrete design examples. As will be shown by Monte-Carlo simulations and EXIT-analysis, the proposed IRA coded IDMA system outperforms a convention- 


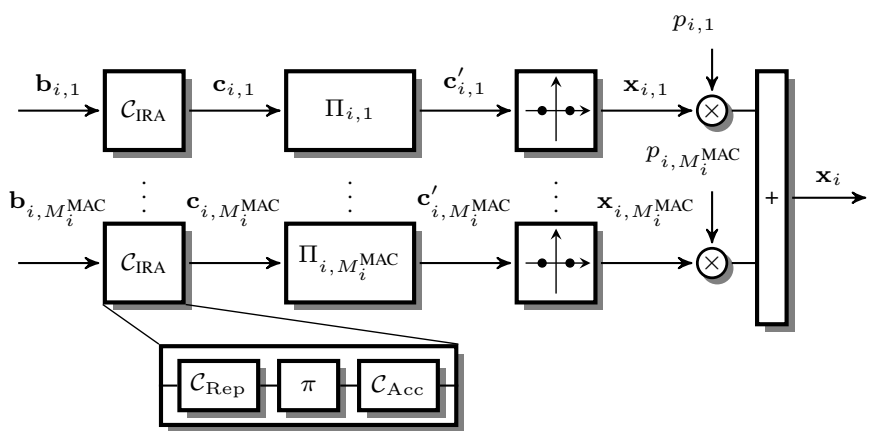

Fig. 2. Transmitter chain at source $i$ consisting of $M_{i}^{\mathrm{MAC}}$ IRA channel encoders $\mathcal{C}_{\text {IRA }}$, source and layer specific interleavers $\prod_{i, m}$ and symbol mappers.

ally coded IDMA system in terms of throughput.

The remainder of this paper is structured as follows. In Sec. II the system model is presented. Sec. III describes the principle IRA code design process. In Sec. IV a concrete design example is given and compared to an IDMA system based on convolutional codes. Finally, Sec. V concludes the paper.

\section{SySTEM Model}

\section{A. Overview}

A topology as depicted in Fig. 1 is considered. $K$ communication pairs communicate simultaneously, aided by a common relay $R$. Only the nodes $A_{k}$ and $B_{k}$ within one pair $k$ are interested in each others messages, i.e., $A_{k}$ is interested in the message of $B_{k}$ and $B_{k}$ is interested in the message of $A_{k}$. The channel between node $i, i \in\left\{A_{1}, B_{1}, \cdots, A_{K}, B_{K}\right\}$ and $R$ is given by $h_{i}=d_{i}^{-\epsilon / 2}$ where $d_{i}$ is the distance between node $i$ and $R$ and $\epsilon$ is the path-loss exponent. Furthermore, each receiving node experiences complex-valued additive white gaussian noise (AWGN) $\mathbf{n}$ of power $\sigma_{\mathrm{n}}^{2}$. Due to the half-duplex constraint, the transmission time can be divided into two transmission phases. In the Multiple Access Phase (MAC), all $2 K$ sources simultaneously broadcast their information to the relay and in the Broadcast Phase $(B C)$, the relay simultaneously forwards the processed information to all $2 K$ nodes $i$.

\section{B. Multiple Access Phase (MAC)}

In the MAC phase, all $2 K$ nodes $i$ simultaneously transmit their messages to the relay $R$ applying Multi-Layer InterleaveDivision Multiple-Access (ML-IDMA) [5] [10]. Each node $i$ transmits $M_{i}^{\mathrm{MAC}}$ layers in parallel. For a fixed code rate, the number of layers directly determines the data rate of node $i$. The transmitter structure of node $i$ is depicted in Fig. 2 . The $M_{i}^{\mathrm{MAC}}$ binary information sequences $\mathbf{b}_{i, m} \in \mathbb{F}_{2}^{N_{\mathrm{b}}}, 1 \leq$ $m \leq M_{i}^{\mathrm{MAC}}$ of length $N_{\mathrm{b}}$ are encoded with the same Irregular Repeat Accumulate (IRA) code of rate $R_{\mathrm{c}}^{\mathrm{MAC}}$ consisting of an outer irregular repetition code $\mathcal{C}_{\text {Rep }}$ and an inner accumulator $\mathcal{C}_{\text {Acc }}$. The code is also the same for all nodes $i$. The resulting binary code sequences $\mathbf{c}_{i, m} \in \mathbb{F}_{2}^{N_{\mathrm{c}}^{\mathrm{MAC}}}$ of length $N_{\mathrm{c}}^{\mathrm{MAC}}$ are interleaved by node and layer specific interleavers $\Pi_{i, m}$. The interleaved code sequences $\mathbf{c}_{i, m}^{\prime}$ are mapped to the BPSK

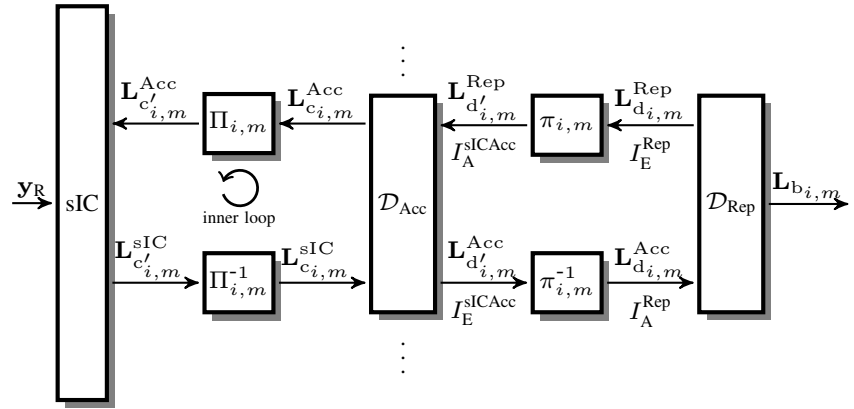

Fig. 3. Receiver chain at the relay consisting of soft interference cancellation (sIC) and $M^{\mathrm{MAC}}$ parallel layer specific decoding branches each consisting of convolutional decoder $\left(\mathcal{D}_{\text {Acc }}\right)$ and repetition decoder $\left(\mathcal{D}_{\text {Rep }}\right)$. Shown is only a single decoding branch.

alphabet $\mathcal{A}=\{+1,-1\}$. Finally, all symbol sequences are scaled by a layer specific factor $p_{i, m} \in \mathbb{C}$ and multiplexed, resulting in the transmit sequence $\mathbf{x}_{i} \in \mathbb{C}^{N_{\mathrm{x}}^{\mathrm{MAC}}}$ of node $i$ as

$$
\mathbf{x}_{i}=\sum_{m=1}^{M_{i}^{\mathrm{MAC}}} p_{i, m} \mathbf{x}_{i, m} .
$$

The scaling factor $p_{i, m}$ consists of a layer specific amplitude factor $\alpha_{i, m}$ and layer specific phase factor $\phi_{i, m}$ as

$$
p_{i, m}=\sqrt{\alpha_{i, m}} e^{j \phi_{i, m}} .
$$

It is chosen such that the overall power constraint $P_{i}$ of node $i$ is met and all layers of node $i$ are uniformly distributed on the unit circle. The latter aims at minimizing the interlayer interference [10]. Furthermore, a node specific random phase factor $\psi_{i} \in\left[-\frac{\pi}{2}, \frac{\pi}{2}\right)$ is introduced in order to reduce the interference among different nodes. The scaling factor then reads

$$
p_{i, m}=\sqrt{\frac{P_{i}}{M_{i}^{\mathrm{MAC}}}} e^{j\left(\frac{m-1}{M_{i}^{\mathrm{MAC}} \pi+\psi_{i}}\right)} .
$$

The transmit signals $\mathbf{x}_{i}$ from all nodes are broadcast simultaneously to the relay resulting in the received signal $\mathbf{y}_{\mathrm{R}}$ at $R$ as

$$
\mathbf{y}_{\mathrm{R}}=\sum_{i} h_{i} \mathbf{x}_{i}+\mathbf{n}_{\mathrm{R}},
$$

where $h_{i}$ is the channel coefficient from node $i$ to $R$ and $\mathbf{n}_{\mathrm{R}}$ is additive white Gaussian noise of power $\sigma_{\mathrm{n}}^{2}$. In total, the received signal consists of $M^{\mathrm{MAC}}=\sum_{i=1}^{2 K} M_{i}^{\mathrm{MAC}}$ layers. The goal at the relay is to recover all information sequences $\mathbf{b}_{i, m}$ from all nodes. Note that it is not sufficient to recover the code sequences $\mathbf{c}_{i, m}$ as a different IRA code will be applied in the broadcast phase. The receiver chain of the relay is given in Fig. 3. It consists of a soft Interference Cancellation (sIC) and $M^{\mathrm{MAC}}$ layer-specific A-Posteriori-Probability (APP) decoding branches. Due to clearness only the decoding branch for an arbitrary layer $m$ of node $i$ is depicted in the figure.

For the sIC different strategies are possible. The optimal sIC follows the Maximum A-Posteriori-Probability (MAP) 
criterion as described in [11]. The MAP detector delivers LogLikelihood Ratios (LLRs) $\mathbf{L}_{\mathbf{c}_{i, m}}^{\text {IC }}$ of the code bits of all layers as

$$
L_{\mathrm{c}_{i, m}}^{\mathrm{IC}}(l)=\log \left(\frac{\sum_{c_{i, m}(l)=0} p(y(l) \mid c(l)) \Pi_{i, \bar{m}} \mathrm{P}\left(c_{i, \bar{m}}(l)\right)}{\sum_{c_{i, m}(l)=1} p(y(l) \mid c(l)) \Pi_{i, \bar{m}} \mathrm{P}\left(c_{i, \bar{m}}(l)\right)}\right)
$$

where $l$ denotes the $l$-th bit and $i, \bar{m}$ means every but the $m$-th layer of node $i$. Eq. (5) has to be carried out for every code bit $c_{i, m}(l)$ across all $M^{\mathrm{MAC}}$ layers and is, thus, very complex even for a reasonable number of nodes and layers.

A different strategy for the sIC is the so-called soft-RAKE (SR) detection [5]. The main simplification compared to the MAP-detection is the assumption that sufficiently many layers are transmitted and, hence, the multi-layer interference can be assumed to be Gaussian distributed according to the central limit theorem. This assumption significantly reduces the detection complexity. For a detailed description of the softRAKE detection refer to [5]. In the following, both strategies are considered and compared to each other.

After sIC the LLRs $\mathbf{L}_{\mathrm{c}_{i, m}}^{\mathrm{IC}}$ of the code bits are fed to the channel decoder which consists of a decoder $\mathcal{D}_{\text {Acc }}$ for the accumulator, carried out as convolutional decoder based on the BCJR algorithm [12], and a decoder for the irregular repetition code $\mathcal{D}_{\text {Rep }}$. The overall detection and decoding procedure is described in detail in Sec. III-B. Finally, the decoding process delivers LLRs $\mathbf{L}_{\mathrm{b}_{i, m}}$ of the information sequences $\mathbf{b}_{i, m}$.

\section{Broadcast Phase (BC)}

In the BC phase the hard estimated source messages $\hat{\mathbf{b}}_{i, m}=$ $\mathcal{Q}\left(\mathbf{L}_{\mathrm{b}_{i, m}}\right)$ are combined in order to reduce the load on the second hop. Adopting the idea of network coding (NC) the $m$-th layers of the two nodes $A_{k}$ and $B_{k}$ within a pair $k$ are combined as

$$
\mathbf{b}_{k, m}^{\mathrm{R}}=\hat{\mathbf{b}}_{A_{k}, m} \oplus \hat{\mathbf{b}}_{B_{k}, m} .
$$

For symmetric rate requirements within a pair, i.e., $M_{A_{k}}^{\mathrm{MAC}}=$ $M_{B_{k}}^{\mathrm{MAC}}$ this leads to $M_{k}^{\mathrm{BC}}=M_{A_{k}}^{\mathrm{MAC}}=M_{B_{k}}^{\mathrm{MAC}}$ relay layers per pair $k$ for the $\mathrm{BC}$ phase. For asymmetric rate requirements, i.e., $M_{A_{k}}^{\mathrm{MAC}} \neq M_{B_{k}}^{\mathrm{MAC}}$, the remaining layers are not combined, leading to $M_{k}^{\mathrm{BC}}=\max \left\{M_{A_{k}}^{\mathrm{MAC}}, M_{B_{k}}^{\mathrm{MAC}}\right\}$ relay layers per pair $k$. The $M^{\mathrm{BC}}=\sum_{k=1}^{K} M_{k}^{\mathrm{BC}}$ relay layers of all pairs $k$ are processed similar to the source processing, i.e., they are encoded with a second IRA code of rate $R_{\mathrm{c}}^{\mathrm{BC}}$ leading to the code messages $\mathbf{c}_{k, m}^{\mathrm{R}} \in \mathbb{F}_{2}^{N_{\mathrm{c}}^{\mathrm{BC}}}$. They are interleaved by a layer and source pair specific interleaver $\Pi_{k, m}^{\mathrm{R}}$, mapped to the BPSK alphabet $\mathcal{A}$ and scaled by a layer specific factor $p_{k, m}^{\mathrm{R}}$ to ensure the overall power contraint $P_{\mathrm{R}}$ of the relay and a uniform phase distribution on the unit circle, i.e., $p_{k, m}^{\mathrm{R}}=\sqrt{\frac{P_{\mathrm{R}}}{M^{\mathrm{BC}}}} e^{j \frac{m-1}{M^{\mathrm{BC}}} \pi}$. The later again ensures minimal inter-layer interference. Finally, all layers are summed up, resulting in the relay transmit signal

$$
\mathbf{x}_{\mathrm{R}}=\sum_{k=1}^{K} \sum_{m=1}^{M_{k}^{\mathrm{BC}}} p_{k, m}^{\mathrm{R}} \mathbf{x}_{k, m}
$$

and broadcast to all nodes. The received signal at node $i$ is then given by

$$
\mathbf{y}_{i}=h_{i} \mathbf{x}_{\mathrm{R}}+\mathbf{n}_{i}
$$

Now, at node $i$ an iterative detection process similar to the detection process at the relay is invoked delivering hard estimates $\hat{\mathbf{b}}_{k, m}^{\mathrm{R}}$ of the relay messages $\mathbf{b}_{k, m}^{\mathrm{R}}$. The desired information is then recovered by substracting the known own information $\mathbf{b}_{i, m}$ in the Galois field $\mathbb{F}_{2}$, i.e.,

$$
\begin{aligned}
& \hat{\mathbf{b}}_{A_{k}, m}=\hat{\mathbf{b}}_{k, m}^{\mathrm{R}} \oplus \mathbf{b}_{B_{k}, m} \\
& \hat{\mathbf{b}}_{B_{k}, m}=\hat{\mathbf{b}}_{k, m}^{\mathrm{R}} \oplus \mathbf{b}_{A_{k}, m}
\end{aligned}
$$

at node $B_{k}$ and $A_{k}$, respectively. For asymmetric rate requirements the layers without network coding obviously directly deliver the desired information.

\section{SySTEM OPTIMIZATION}

\section{A. Overview}

Under the assumption of error-free transmission, the maximum spectral efficiency $\eta_{\max }$ of the overall transmission is given by

$$
\eta_{\max }=M^{\mathrm{MAC}} R_{\mathrm{c}}^{\mathrm{MAC}} \frac{T_{\mathrm{MAC}}}{T_{\mathrm{MAC}}+T_{\mathrm{BC}}}
$$

where $T_{\mathrm{MAC}}$ and $T_{\mathrm{BC}}$ are the durations of MAC and $\mathrm{BC}$ phase, respectively. Since the number of information bits per layer $N_{\mathrm{b}}$ is the same in both phases, the durations of both phases only depend on the code rates $R_{\mathrm{c}}^{\mathrm{MAC}}$ and $R_{\mathrm{c}}^{\mathrm{BC}}$. Eq. (10) can, therefore, be written as

$$
\eta_{\max }=M^{\mathrm{MAC}} \frac{R_{\mathrm{c}}^{\mathrm{MAC}} R_{\mathrm{c}}^{\mathrm{BC}}}{R_{\mathrm{c}}^{\mathrm{MAC}}+R_{\mathrm{c}}^{\mathrm{BC}}} .
$$

Thus, aiming at a high spectral efficiency, thorough design of the channel codes is essential. This section illustrates the principal design process of the IRA code in the context of the considered multi-pair bidirectional relaying system. The goal of this design process is to find weighting factors for the different component repetition codes in order to achieve convergence at the desired working point.

Principally, the design process consists of two parts. First, the transfer characteristic of the inner detection loop consisting of sIC and convolutional decoder $\mathcal{D}_{\text {Acc }}$ has to be determined. Since the characteristic of both components cannot be described analytically, it has to be evaluated numerically [9]. The same clearly holds also for the combination of both components. The second part is the optimization of the component repetition codes to fit the characteristic of the inner detection loop determined in the first phase. Due to the network coding at the relay, the load in the $\mathrm{BC}$ phase is usually lower than the load in the MAC phase. Therefore, code design should be performed separately for both phases. 


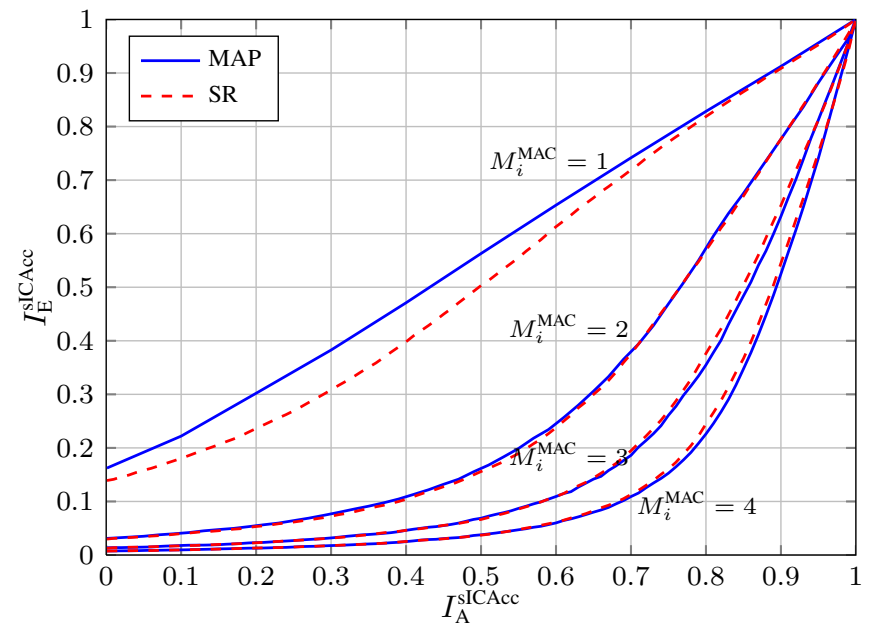

Fig. 4. Transfer characteristic of the inner detection loop for $2 K=4$ nodes with $P_{i}=1$ and different numbers of layers per node $M_{i}$ at $1 / \sigma_{\mathrm{n}}^{2}=0 \mathrm{~dB}$ $N_{\mathrm{it}, \mathrm{i}}=3$ inner iterations between detector and accumulator for MAP; $N_{\mathrm{it}, \mathrm{i}}=$ 5 inner iterations for soft-RAKE (SR).

\section{B. Code Design}

1) EXIT-analysis of inner loop: The inner detection loop consists of sIC and convolutional decoder based on the BCJR algorithm. Since the transfer characteristics of both components cannot be described in analytical form, they have to be determined numerically. The transfer function $T$ [7] of the inner loop, which describes the relation between the a-priori mutual information $I_{\mathrm{A}}^{\mathrm{sICAcc}}$ from the outer irregular repetition code and the extrinsic mutual information $I_{\mathrm{E}}^{\text {sICAcc }}$, is given by

$$
I_{\mathrm{E}}^{\mathrm{sICAcc}}=\mathrm{T}\left(I_{\mathrm{A}}^{\mathrm{sICAcc}}, M^{\mathrm{MAC}},\left\{P_{i}\right\}, \sigma_{\mathrm{n}}^{2}\right), \quad 0 \leq I_{\mathrm{A}}^{\mathrm{sICAcc}} \leq 1 .
$$

It depends on the working point of the system, i.e., the overall number of transmitted layers $M^{\mathrm{MAC}}$, the transmit powers of all nodes $\left\{P_{i}\right\}$ and the noise variance $\sigma_{\mathrm{n}}^{2}$.

Fig. 4 exemplarily shows the transfer characteristics for the inner detection loop for MAP as well as for SR detection. For the MAP-based detection loop $N_{\mathrm{It}}^{\text {in }}=3$ inner iterations and for the SR-based detection $N_{\text {It }}^{\text {in }}=5$ inner iterations were performed, as the latter usually requires more iterations to converge. Additional iterations do not improve the performance any further. As can be seen in the figure, the MAP detection leads to a slighly better performance than the softRAKE detection for a small number of transmitted layers $\left(M_{i}^{\mathrm{MAC}}=1\right)$. This is due to the inherent assumption for the SR detection, that all multi-layer interference is approximately Gaussian distributed and, hence, is modelled as such. For a small number of layers this assumption clearly is not true, leading to the observed performance loss compared to the MAP detection. For higher numbers of layers, however, both detectors lead to almost the same performance. Since the computational complexity of the SR is much lower compared to the complexity of the MAP detector, the following investigations will be done based on SR detection.

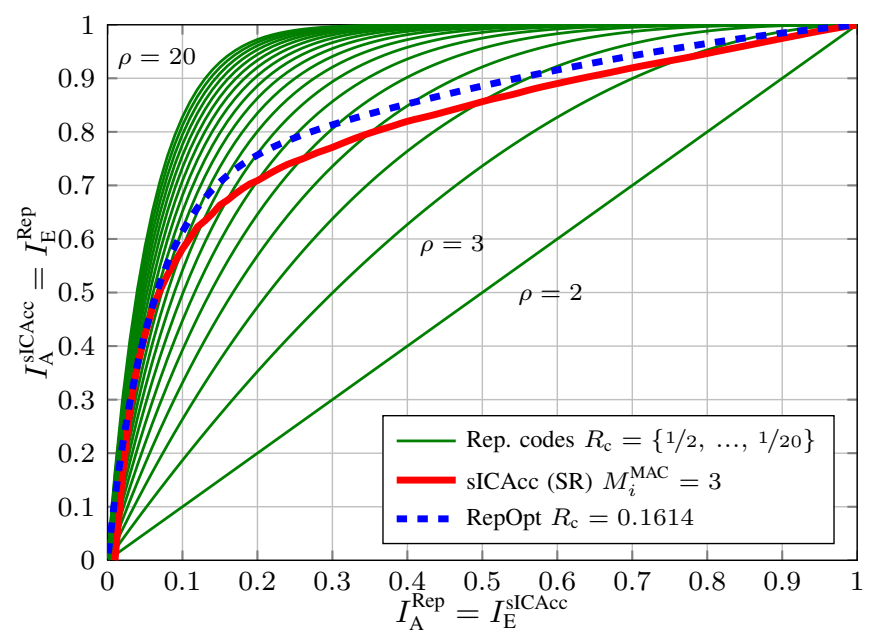

Fig. 5. Optimization of repetition factors $w_{\rho}$ to transfer characteristic of inner detection loop. $2 K=4$ nodes with $P_{i}=1$ and $M_{i}^{\mathrm{MAC}}=3$ layers per node. $1 / \sigma_{\mathrm{n}}^{2}=0 \mathrm{~dB}, R_{c}^{\mathrm{MAC}}=0.1614$.

2) Adaptation of outer code: The outer code is an irregular repetition code, i.e., a mixture of component repetition codes. The transfer characteristic of each component code as well as the code mixture can be described in analytical form. The transfer characteristic of a repetition code of rate $R_{\mathrm{c}}=\frac{1}{\rho}$ is given as

$$
I_{\mathrm{E}, \rho}^{\mathrm{Rep}}=\mathrm{T}_{\rho}\left(I_{\mathrm{A}, \rho}^{\mathrm{Rep}}, \rho\right)=\mathcal{J}\left((\rho-1) \mathcal{J}^{-1}\left(I_{\mathrm{A}, \rho}^{\mathrm{Rep}}\right)\right),
$$

where

$$
\begin{aligned}
\mathcal{J}(\nu) & =\left(1-2^{-1.0605 \nu^{0.8935}}\right)^{1.1064} \\
\mathcal{J}^{-1}(\nu) & =\left(-1 / 1.0605 \log _{2}\left(1-\nu^{1 / 1.1064}\right)\right)^{1 / 0.8935}
\end{aligned}
$$

denote the J-function and its inverse. The transfer characteristic of the code mixture is then given as

$$
I_{\mathrm{E}}^{\mathrm{Rep}}=\sum_{\rho=\rho_{\min }}^{\rho_{\max }} w_{\rho} I_{\mathrm{E}, \rho}^{\mathrm{Rep}},
$$

where $0 \leq w_{\rho} \leq 1$ is the weight of the repetition code of rate $R_{\mathrm{c}}=\frac{1}{\rho}$ in the mixture. The goal is now to match the transfer characteristic $I_{\mathrm{E}}^{\text {Rep }}$ of the irregular repetition code to match the characteristic $I_{\mathrm{A}}^{\text {sICAcc }}$ of the inner block by adapting the weights $w_{\rho}$, i.e,

$$
\begin{aligned}
& {\left[w_{\rho_{\min }}, \ldots, w_{\rho_{\max }}\right] }=\arg \min \left\{I_{\mathrm{E}}^{\mathrm{Rep}}-I_{\mathrm{A}}^{\mathrm{sICAcc}}+\Delta\right\} \\
& \text { s.t. } \sum_{\rho=\rho_{\min }}^{\rho_{\max }} w_{\rho}=1 \\
& I_{\mathrm{E}}^{\mathrm{Rep}}>I_{\mathrm{A}}^{\mathrm{sICAcc}},
\end{aligned}
$$

where $\Delta$ is the minimal allowed gap between both transfer curves and mainly determines the number of required iterations to achieve convergence. 


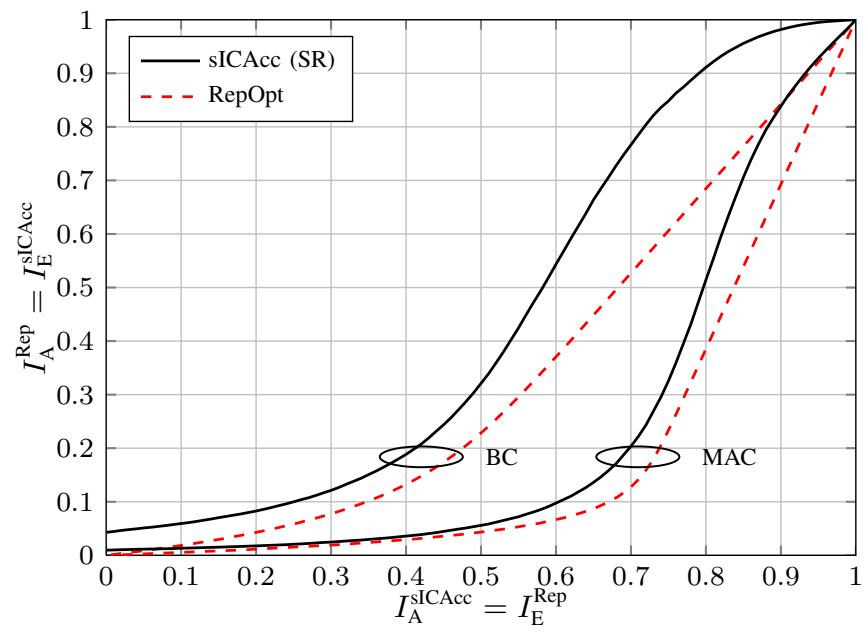

Fig. 6. Transfer characteristics for inner detection loop and outer irregular repetition code for MAC and BC phase. Four nodes with $P_{i}=1$ and $M_{i}=4$ layers per node and relay with $P_{\mathrm{R}}=4.1 / \sigma_{\mathrm{n}}^{2}=5 \mathrm{~dB}, R_{\mathrm{c}, 1}=0.1826$, $R_{\mathrm{c}, 2}=0.3386$

Fig. 5 illustrates the code adaption. The red curve sICAcc exemplarily denotes the transfer characteristic of the inner block for $M_{i}^{\mathrm{MAC}}=3$ layers per node $i$, which was determined numerically in Fig. 4. The green curves represent the transfer characteristics of the repetition codes of rates $R_{\mathrm{c}}=\frac{1}{\rho}$ for $2 \leq \rho \leq 20$. The blue curve RepOpt shows the transfer characteristic of the resulting optimized irregular repetition code for $\rho_{\min }=2$ and $\rho_{\max }=20$ and $\Delta=10^{-2}$ after numerical evaluation of eq. (16a).

The code rate of the resulting irregular repetition code is then given as the sum of the code rates of the component codes weighted by the corresponding weighting factors as

$$
R_{\mathrm{c}}=\sum_{\rho=\rho_{\min }}^{\rho_{\max }} \frac{w_{\rho}}{\rho} .
$$

Since the accumulator is a rate one code, (17) is also the code rate of the overall IRA code.

\section{DeSIGN EXAMPLE}

\section{A. Symmetric rate requirements}

In the following, the two IRA codes for MAC and BC phase for a system with $2 K=4$ nodes and one common relay as depicted in Fig. 1 are designed. All nodes have the same power constraint $P_{i}=1$ and the same distance $d_{i}=1$ to the relay. The transmit power of the relay is set to $P_{\mathrm{R}}=4$. Furthermore, all nodes have the same rate requirements and, therefore, should transmit the same number of layers $M_{i}^{\mathrm{MAC}}$. Thus, the overall number of layers in the MAC phase is restricted to $M^{\mathrm{MAC}} \in\{4,8,12,16, \ldots\}$ and the corresponding number of layers in the $\mathrm{BC}$ phase is restricted to $M^{\mathrm{BC}} \in\{2,4,6,8, \ldots\}$. All layers of one source equally share the source's transmit power. In order to achieve a fair comparison to a conventional code combination for IDMA, i.e., a serial concatenation of a convolutional code and a repetition code, the working point is set to $1 / \sigma_{\mathrm{n}}^{2}=5 \mathrm{~dB}$ and the number of layers per source is
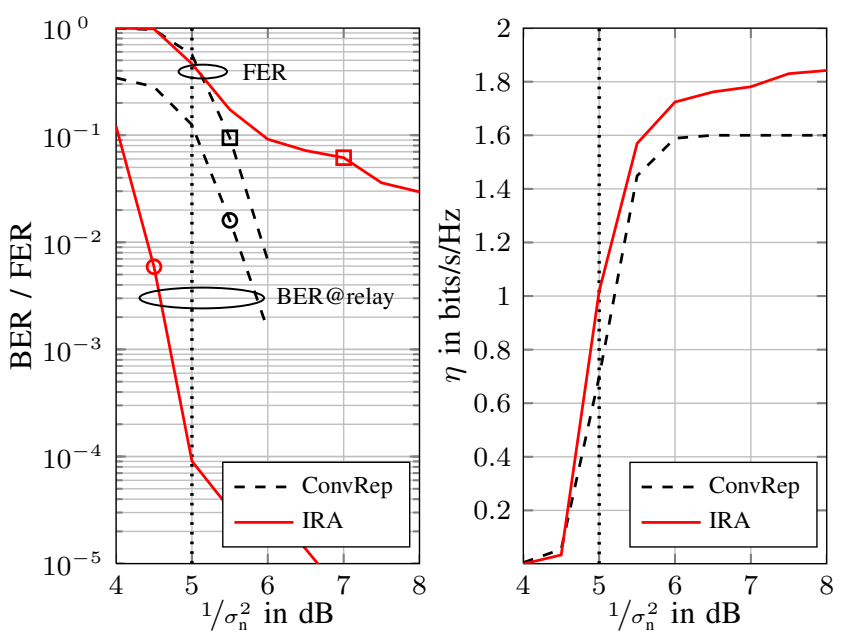

Fig. 7. Average bit error rate BER (o) at the relay and frame error rate FER ( $\square$ ) at the receiving nodes. Corresponding system throughput $\eta$ for IRA coded IDMA and convolutionally coded IDMA. $P_{i}=1, M_{i}^{\mathrm{MAC}}=4, P_{\mathrm{R}}=4$, $R_{\mathrm{c}}^{\mathrm{MAC}}=0.1826, R_{\mathrm{c}}^{\mathrm{BC}}=0.3386$.

set to $M_{i}^{\mathrm{MAC}}=4$. At this point the combination of the nonsystematic, non-recursive $(5 ; 7)_{8}$ convolutional code of rate $R_{\mathrm{c}, \text { Conv }}=1 / 2$ and the repetition code of rate $R_{\mathrm{c}, \mathrm{Rep}}=1 / 3$ converge as can be seen in Fig. 7. The frame length is set to $N_{\mathrm{c}}^{\mathrm{MAC}}=10^{4}$ code bits. In the BC phase the code rate of the repetition code is increased to $R_{\mathrm{c}, \mathrm{Rep}}=1 / 2$ as the number of layers is reduced due to network coding.

Fig. 6 depicts the transfer characteristic of the inner loop (sICAcc) consisting of SR detector and convolutional decoder for the given working point for overall $M^{\mathrm{MAC}}=16$ layers (MAC phase) and $M^{\mathrm{BC}}=8$ layers (BC phase). In total $N_{\mathrm{It}}^{\mathrm{in}}=$ 5 inner iterations between SR detector and convolutional decoder were performed. Also given are transfer characteristics of the optimized irregular repetition codes (RepOpt) for both phases. Note that there is still a significant gap between the corresponding transfer characteristics as the goal of the optimization was to achieve convergence with a resonable amount of iterations. For both systems, i.e., the IRA coded system as well as the convolutionally coded IDMA system the number of (outer) iterations was set to $N_{\mathrm{It}}^{\text {out }}=25$.

In Fig. 7 on the left hand side the average bit error rate BER at the relay as well as the average frame error rate FER at the receiving nodes for both code combinations are given. As can be seen from the BER, convergence for the IRA coded system starts around $1 / \sigma_{\mathrm{n}}^{2}=4 \mathrm{~dB}$, approximately $1 \mathrm{~dB}$ before the working point. This is mainly due to the rather conservative code design. The conversion for the convolutionally coded system starts around $1 / \sigma_{\mathrm{n}}^{2}=5 \mathrm{~dB}$. Although the IRA code leads to a steeper slope in the waterfall region compared to the convolutional code, it shows the typical error floor behaviour below BER $=10^{-4}$ which leads to an only moderate decrease in FER.

Under the assumption of a selective repeat hybrid ARQ protocol the throughput $\eta$ is given depending on the frame error rate FER as 


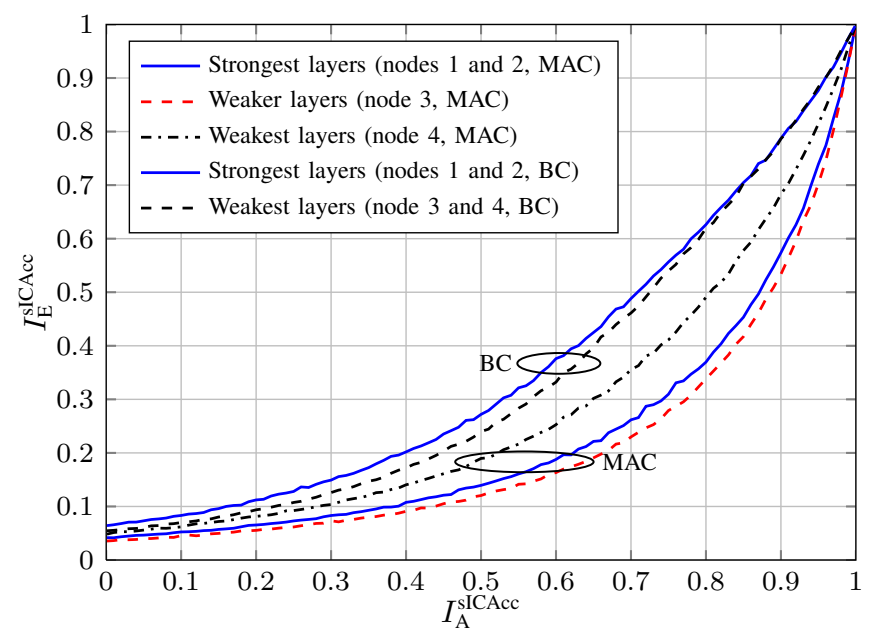

Fig. 8. Transfer characteristic of the inner detection loop for four nodes with $P_{i}=1$ and different numbers of layers per node $M_{i}$ at $1 / \sigma_{\mathrm{n}}^{2}=5 \mathrm{~dB}$. $N_{\mathrm{it}, \mathrm{i}}=5$ inner iterations between detector and accumulator.

$$
\eta=M^{\mathrm{MAC}} \frac{R_{\mathrm{c}}^{\mathrm{MAC}} R_{\mathrm{c}}^{\mathrm{BC}}}{R_{\mathrm{c}}^{\mathrm{MAC}}+R_{\mathrm{c}}^{\mathrm{BC}}}(1-\mathrm{FER}) .
$$

On the right hand side of Fig. 7 the throughput $\eta$ is plotted for both systems. Despite the moderate decrease in FER the IRA coded system clearly outperforms the convolutionally coded system due to the higher code rates during both transmission phases. The IRA system achieves an increase in throughput of approximately $0.2 \mathrm{bit} / \mathrm{s} / \mathrm{Hz}$ for the given scenario and working point.

\section{B. Asymmetric rate requirements}

The same system as for the symmetric case is considered. However, the rate requirements of the nodes are now such that both nodes of the first pair each transmit two layers, i.e., $M_{1}^{\mathrm{MAC}}=M_{2}^{\mathrm{MAC}}=2$, and the nodes of the second pair transmit four and six layers, respectively, i.e., $M_{3}^{\mathrm{MAC}}=4$ and $M_{4}^{\mathrm{MAC}}=6$. Due to the per node power constraint, the power of the layers of different nodes are not equal anymore. Thus, in order to optimize the channel code, the layers of different powers have to be considered individually. Since the transfer characteristic of each layer depends on the transfer characteristics of all other layers which are not equal, EXITanalysis gets difficult. In order to simplify this task, it is assumed that stronger layers, i.e., those with higher power, are decoded successfully before weaker layers. That means for layers of a certain power stronger layers can be cancelled perfectly while weaker layers are completely unknown, i.e. fully intefere. Fig. 8 depicts the transfer characteristics of the different layers.

From the transfer characteristics it can be seen that different IRA codes should be designed for different layers. Fig. 9 depicts results from Monte-Carlo simulations of the given system. As can be seen, node 3 achieves approx. twice the throughput as nodes 1 and 2 and node 4 achieves three times the throughput, as initially desired.
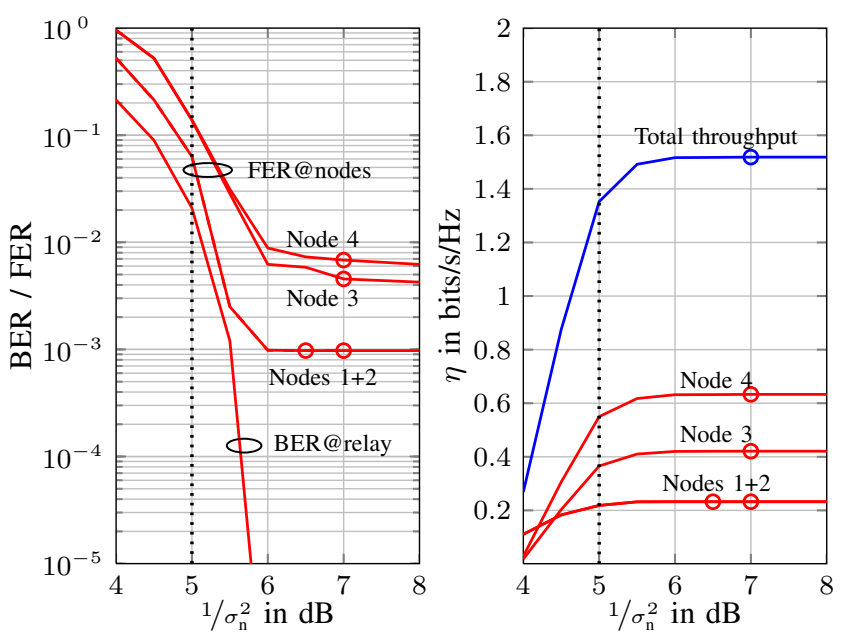

Fig. 9. Average bit error rate BER at the relay and frame error rates FER at the receiving nodes. Corresponding total system throughput $\eta$ and individual throughputs. $P_{i}=1, M_{1}^{\mathrm{MAC}}=M_{2}^{\mathrm{MAC}}=2, M_{3}^{\mathrm{MAC}}=4, M_{4}^{\mathrm{MAC}}=6$, $P_{\mathrm{R}}=4$.

\section{CONCLUSION}

In this paper we designed Irregular Repeat Accumulate (IRA) codes for IDMA-based multi-pair bidirectional relaying systems. We presented the principle design process and gave concrete design examples for symmetric as well as for asymmetric rate requirements. As was shown by numerical evaluations, the IRA coded system clearly outperformed the conventionally coded IDMA system based on convolutional codes in terms of throughput.

\section{REFERENCES}

[1] S. Zhang, S. Liew, and P. Lam, "Hot Topic: Physical Layer Network Coding," in Proc. International Conference on Mobile Computing and Networking (MobiCom), Los Angeles, CA, USA, 2006, pp. 358-365.

[2] R. Ahlswede, M. Cai, S.-Y. Li, and R. Yeung, "Network Information Flow," IEEE Transactions on Information Theory, vol. 46, no. 4, pp. 1204-1216, Jul. 2000.

[3] C. Fragouli, J. Boudec, and J. Widmer, "Network Coding: An Instant Premier," ACM SIGCOMM Computer Communication Review, vol. 36, no. 1, pp. 63-68, Mar. 2006.

[4] M. Chen and A. Yener, "Multiuser Two-Way Relaying for Interference Limited Systems," in IEEE International Conference on Communications (ICC 2008), Beijing, China, May 2008.

[5] L. Ping, L. Liu, K. Wu, and W. Leung, "Interleave-Division MultipleAccess," IEEE Transactions on Wireless Communications, vol. 5, no. 4, pp. 938-947, Apr. 2006.

[6] K. Kusume, G. Bauch, and W. Utschick, "IDMA vs. CDMA: Analysis and Comparison of Two Multiple Access Schemes," IEEE Transactions on Wireless Communications, vol. 11, no. 1, pp. 78-87, Jan. 2012.

[7] S. ten Brink, "Convergence of Iterative Decoding," IEEE Electronic Letters, vol. 35, no. 13, pp. 1117-1119, May 1999.

[8] H. Jin, A. Khandekar, and R. McEliece, "Irregular Repeat Accumulate Codes," in Second International Symposium on Turbo Codes, Brest, France, Sep. 2000.

[9] S. ten Brink and G. Kramer, "Design of Repeat-Accumulate Codes for Iterative Detection and Decoding," IEEE Transactions on Signal Processing, vol. 51, no. 11, pp. 2764-2772, Nov. 2003.

[10] P. A. Höher, H. Schöneich, and J. C. Fricke, "Multi-layer interleavedivision multiple-access: theory and practise," European Transactions on Telecommunications, vol. 19, pp. 523-536, Jan. 2008.

[11] M. Noemm, T. Wo, and P. A. Höher, "Mutilayer APP detection for IDM,” Electronic Letters, vol. 46, no. 1, pp. 96-97, Jan. 2010.

[12] L. Bahl, J. Cocke, F. Jelinek, and J. Raviv, "Optimal Decoding of Linear Codes for Minimizing Symbol Error Rate," IEEE Transactions on Information Theory, vol. 20, no. 2, pp. 284-287, Mar. 1974. 